\title{
An implantable artificial pancreas
}

\author{
W. Schubert \\ P. Baurschmidt \\ J. Nagel \\ R. Thull \\ M. Schaldach
}

Zentralinstitut für Biomedizinische Technik, Friedrich Alexander Universität, Turnstrasse 5, D-8520 Erlangen, West Germany

Keywords-Artificial pancreas, G/ucose sensor, Implantable insulin pump, Piezoelectric dosing valve,
Telecommunication

Abstract-The artificial implantable pancreas is seen as the optimal means of therapy in patients with severe diabetes mellitus. The implantable pancreas consists of three modules; (i) the dosing unit, (ii) the control circuit and (iii) a glucose sensor for the realisation of a feedback system. Intensive research has been devoted to essential items, such as a dosing valve with only minimal susceptibility to interference, the design of an electrochemical sensor for continuous/y monitoring the glucose leve!, and design criteria for data transfer between an external programmer/monitor and the implant.

\section{Introduction}

GENERALLY therapy of diabetes involves one or two injections of depot insulin daily. In this way the blood sugar can be matched only incompletely to normal requirements; quite apart from the discomfort of the daily injection, the inconvenience of having to follow a diet, and the complications of the disease.

Diabetes mellitus is characterised by a relative or an absolute insulin deficiency. If the normal insulin secretion fails, fuel homeostasis is deranged. The earliest sign of the disease is the loss of control of the blood glucose level. Depending upon the duration of diabetes degenerative complications appear which include blindness, heart disease and kidney failure. Improved therapy, especially for younger patients, must include new methods of insulin delivery.

In many laboratories various external devices have been developed as feedback-controlled instruments for the regulation of insulin delivery on a minute-byminute basis in accordance with measured blood glucose level and their rate of change. Despite great efforts in the field of investigation, and despite the fact that several therapeutic possibilities have achieved the goal of ideal blood glucose control, long-term therapy is problematic owing to the size of the apparatus and the blood loss resulting from the need for continuous extracorporeal glucose sensing.

A certain improvement in the therapy has been achieved by portable units that function as open-loop systems without a glucose measuring device. Clinical studies have demonstrated the feasibility of a preprogrammed and/or reprogrammable insulin infusion, in conjunction with daily glucose monitoring.

A prerequisite for further extension of the therapy is the miniaturisation of the portable devices to a size

First received 10th April 1980

$0140-0118 / 80 / 040527+11 \$ 01 \cdot 50 / 0$

(C) IFMBE: 1980 comparable with that of artificial heart pacemakers, and an expected implant lifetime of five years or more. This means that the various components of the artificial pancreas, sensor, microcomputer and delivery pump have to be assembled and hermetically sealed in a long-term stable biocompatible metal housing. Insulin infusion is realised by means of a venous catheter. In combination with a telemetry system, the unit should be reprogrammable to match the parameters to the patient's need if no glucose sensor is available or if an implanted sensor fails. Meanwhile further trials with highly sophisticated portable units should improve our knowledge as to the patient's insulin need in the presence of varying external stresses.

Up to now, implantable pumps for clinical application and suitable for the delivery of insulin have not been developed. However several groups are working on the development of both implantable dosing units and an implantable glucose sensor. Intravascular blood glucose sensing is difficult owing to the complex technology involved, and the foreign-body reaction of blood. The measurement of glucose in tissue would be easier to handle, but it has not been established whether the extravascular tissue concentration of glucose is sufficiently significant to serve as an input signal for a closed-loop system. Only when these questions have been answered and a suitable pumping and dosing unit have been developed, can the closed-loop system for the control of blood glucose be realised and miniaturised for implantation. Advanced technologies developed for programmable heart pacemakers, that is large-scale integrated electronic circuits, needing only a minimum of electrical energy to function, and highly reliable materials for mechanical devices together with hermetically sealed and bicompatible encapsulations, should be able to advance the development of an artificial pancreas in the near future. 


\section{Insulin-delivery systems}

A system for the delivery of drugs by continuous long-term intravascular infusion is a desirable form of therapy for many disease states. However, this form of drug administration has not been feasible to date, owing to problems associated with long-term percutaneous cannulae from external infusion devices and relative patient immobility.

To overcome these problems, a totally implantable infusion pump that can be refilled by percutaneous injection is needed. Dosing systems for an implantable artificial pancreas have to be able to administer with great accuracy very small insulin doses that vary with time. The implant lifetime requirement is at least ten years. In the field of fluid-delivery systems for use in the human body, the present devices are either not totally implantable or not directly controllable. A miniature implantable roller-type pump (SUMmers, 1970 ) has existed since 1970. This pump requires an extracorporeal power source which is in the form of an external motor magnetically coupled to the pump through the skin. Feedback control would require a rather complicated arrangement of the implanted and extracorporeal units. A fully implantable fluiddelivery system was mentioned in 1972 (BLACKSHEAR, et al., 1979). The presently available device does not permit the output to be varied.

The main problem in designing implantable drugdelivery systems is the limited amount of insulin that can be stored within the reservoir. This means that the reservoir must be replenished percutaneously. But this requirement eliminates systems which use irreversible phenomena, such as osmosis, as the driving force, or constant dosing rates effected by means of mechanical components, for example, capillary tubes.

Other requirements that have to be met are biocompatibility of the materials, low power consumption, no wear of moving parts and drug delivery against a back pressure.

In principle, dosing systems comprise an insulin reservoir, a pump and, if required, valves. When designing reservoirs for implantable systems, it must be ensured that no vacuum can occur which would prevent drug delivery.

At present there are two different types of delivery systems at the experimental stage: delivery pumps that require active valve control of the outflow and dosing pumps which generally need valves for control.

\subsection{Delivery pumps}

The widespread vapour-pressure pump is powered by evaporation at a constant pressure in an enclosed liquid/vapour system.

This unit is a membrane pump consisting of two chambers for the stored insulin and the vapour system. The pressure against the membrane will be constant, irrespective of the amount of insulin injected, as long as there is equilibrium between the vapour phase and the fluid phase.

The advantages of the pump may be summarised thus: no parts giving rise to friction and wear; no separate insulin reservoir; no need for electric energy for pumping, and convenient replenishment by using a syringe.

The dosing rate of the pump is determined by two flow restrictors (capillaries) in series, one of which can be bypassed by activating the valve (Fig. 1). This means that the pumping system infuses continuously, the rate of infusion resulting from the length of time the second flow restrictor is bypassed. The rate of infusion is governed by the Hagen-Poiseuille equation and depends on the pressure drop across the capillary tube. This pressure drop is the difference between the pump's internal vapour-pressure and the pressure that exists at the end of the capillary, i.e. the body's central venous pressure, or the atmospheric pressure if pumping is into the tissue. Although the systems described are capable of quantitative delivery of small volumes of insulin, certain disadvantages are obvious. Changes in pressure caused by changes in ambient pressure or by crystallisation of insulin or clotting blood give rise to uncontrollable changes in the infusion rate. Other drawbacks are the high current consumption of solenoid valves, which is incompatible with longterm implantation of up to ten years, and the impossibility of changing the basilar rate after implantation.

\subsection{Dosing pumps}

Precision pumps for drug delivery are undergoing experimental and clinical investigations by different groups. A piezoelectric pump intended for use in an

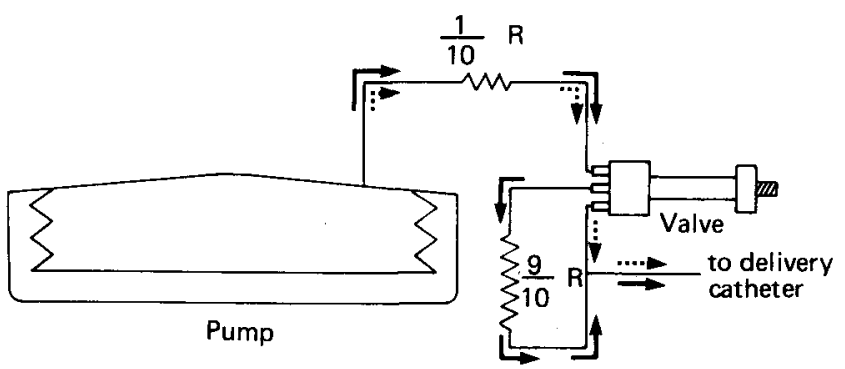

Fig. I Schematic illustration of pump, valve and flow restrictor arrangement (see BLACKSHEAR, 1979)

$--\rightarrow$ flow path with valve activated

$\longrightarrow$ flow path with valve closed 
artificial pancreas was constructed by THOMAs et al. in 1975. In this device pressure is developed by a bellow formed from two opposed piezoelectric disc benders connected to the high-impedance secondary winding of a miniature transformer. Active properly phased valve control is achieved by a miniature solenoid valve connected in series with the transformer primary winding and a rectangular-wave generator. Further development led to a pump (SPENCER et al., 1978) which in addition has piezoelectric valves to reduce power consumption. The piezoelectric pump and valves system has three chambers, an inlet chamber connected to the insulin reservoir, a pump chamber and an outlet chamber connected to the patient's body.

In principle, the piezoelectric pump consists of a short cylinder, the top and bottom of which are made of flexible pieces of metal to which piezoceramic discs are bonded on both sides. When voltage is applied, the piezoelectric discs tend to increase or decrease in size, but because they are bonded to the flexible metal ends of the cylinder a bulging effect is produced. The resulting change in volume of the cylinder provides the pumping effect. The advantages of this pump are the very small energy consumption of the piezoceramic material, and the dosing of definite volumes that are virtually independent of the back pressure. Disadvantages are the need for a special insulin reservoir and for two valves, one between reservoir and pump and the other between pump and catheter (Fig. 2).

The pump valve open/close sequence is as follows. At the start of the pump cycle, the intake valve is opened and the pump is actuated for an intake stroke for $100 \mathrm{~ms}$. The intake valve is then closed and the outlet valve opened. The voltage is removed from the pump, thus returning it to its normal position and providing the insulin to the pump outlet for $200 \mathrm{~ms}$. At this point the outlet valve is closed.

The roller pump (FraneTZKY et al., 1976) does not need valves and it always delivers the same volume, irrespective of the back pressure. On the other hand, the energy consumption of the ulectromotor when the roller pump is activated is high compared with the piezoelectric units, especially in the initial driving phase; furthermore, the system needs an additional insulin reservoir and there is an appreciable risk of destruction of the tube by friction or fulling.

Similar to this unit is another roller pump (ALBISSER et al., 1978) presently in use in a portable insulindelivery system.

Pumping is accomplished by the peristaltic action of an offset rotor driven directly by a 2048:1 reduction gearhead which in turn is mounted on the motor. The insulin solution is carried in a silicone-rubber pump tube, which enters the pumping head and occupies the space between the rotor and the aluminum stator in a single-spiral configuration. The tolerances of the pump components are controlled to within $\pm 25 \mu \mathrm{m}$. This guarantees that, at all positions, the rotor occlusion of the pump tube is appropriate and not excessive.

Although the systems described are capable of quantitative delivery of small volumes of solution, each has certain drawbacks which limit clinical application. Thus there is still a need in the implantation field for a delivery system which is amenable to uncomplicated feedback control, is able to deliver small discrete aliquots safely and reliably, and which has such a low power requirement that a relatively long implantation life is feasable.

A miniature pump which meets these requirements comprises a modified diaphragm pump, driven by a propellant gas and a pumping valve, which is constructed of two titanium shims epoxied on both sides with piezoceramic discs (Fig. 3). The bimorphs and the ceramic disc are assembled in a holder. The holder is integrated into the diaphragm pump. The inner piezocrystals lie close up against a ceramic disc. The function of the valve is characterised by a dosing and a pumping phase. For dosing, the left bimorph in contact with the reservoir opens and the insulin, driven by the pump pressure, fills the space between the membrane and the ceramic disc. Activation of the right and deactivation of the left bimorph causes the insulin dose to pass from the left to the

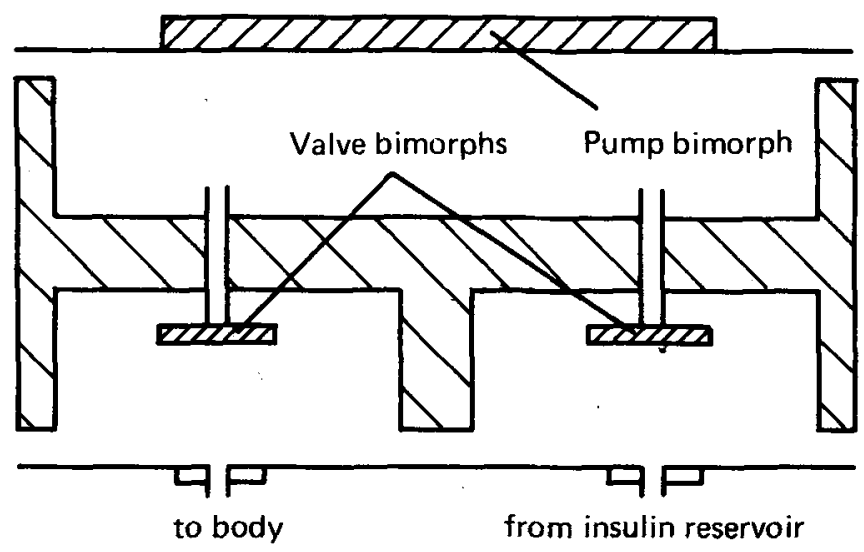

Fig. 2 Diagram (side view) of piezoelectric pump and valves (see SPENCER, 1978) 
right space. In this phase the insulin reservoir is already closed against the biological environment. The expulsion of the insulin is brought about by deactivation of the right bimorph.

In this way a defined volume of insulin is removed from the reservoir, passed through the valve and infused into the body.

The right bimorph can be replaced by a permanent-magnet valve of sufficient power to keep
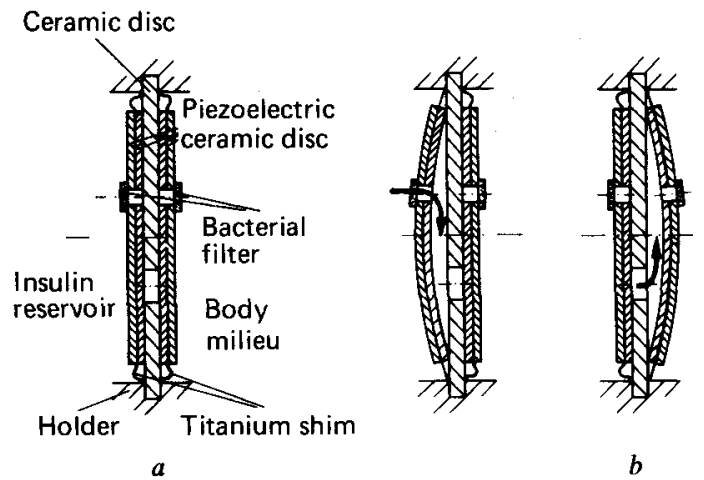

the opening closed against the pump pressure during filling of the dosage chamber, while permitting it to be opened by the force of the deactivated bimorph. The advantages of the pump are:

$(a)$ insulin-compatible material, $(b)$ no electric energy requirement, $(c)$ no moving parts, $(d)$ no extra insulin reservoir and $(e)$ low weight. The main part of the dosing valve is a piezoceramic equipped unit. This kind of valve has the advantages of: (i) low energy consumption, (ii) easy to handle control and (iii) accurate delivery of small amounts of insulin.

\subsection{Pump control}

The insulin-delivery system comprising piezoelectric pump and valves is designed around a 24-hour clock that can be programmed to deliver insulin in varying amounts on a regular basis. In the normal program mode, the unit would deliver insulin at high rates following meal periods and would decrease its insulin delivery rates between meals, during periods of exercise, and during sleep periods. The timer and control logic packages are designed to operate from a $5 \mathrm{~V}$ power supply. The pump and valve system require a higher voltage and this is provided by a pulse transformer and driver circuit.

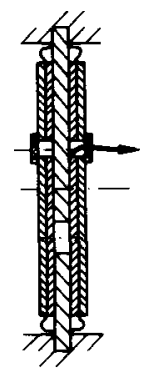

Fig. 3 Schematic illustration of pumping valve
(a) structure
(b) operation

To increase the safety of the implantable infusion system, the control of the piezoelectric pumping valve (SCHUBERT et al., 1979) is realised by a system operating on three levels with decreasing priority. Reliable operation is guaranteed by a data check performed at each level for the data coming from the next higher level.

1st level: When the sensor is working, the included control circuit transmits impulse bursts to the control unit which are proportional to the actual blood glucose level. After signal checking, the control unit calculates the amount of insulin to be infused, on the basis of patient-specific parameters, recorded in the program memory containing the control algorithm. The correct amount is coded again by means of a pulse burst and then transmitted to the dosing unit.

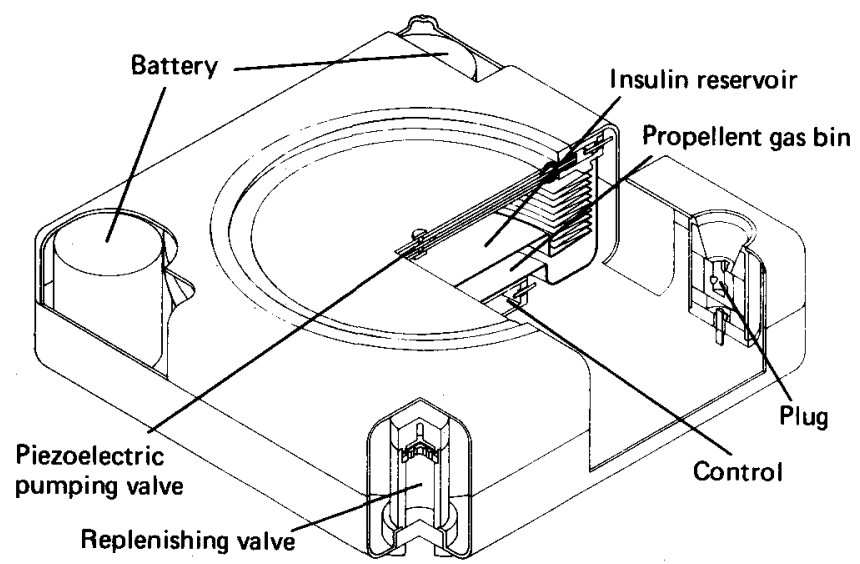

Fig. 4 Implantable dosing unit 
Each checked pulse represents a constant opening period of the valve.

2nd level: If no sensor is used, or if the sensor employed fails, the calculation of the amount of insulin occurs on the basis of profiles obtained in a preimplantation phase with the aid of artificial bedside pancreas. These profiles are recorded in a program memory containing the daily infusion rate. The system includes a facility for changing the data if necessary. A clock in the control unit guarantees that the scanning of the profile is carried out at the right time. Communication with the dosing unit is effected by pulse bursts. Both the daily infusion rate program and the control algorithm of level 1 and level 2 can be programmed by means of a telemetry system.

3rd level: If no bursts have been received by the dosing unit or, if on checking, the transmitted signals are found to be incorrect, the dosing unit feeds a constant basilar rate triggered by the case of need control. The dosing unit is designed as a hermetically sealed metallic can having outside dimensions of $66 \times 66 \times 20 \mathrm{~mm}$ (Fig. 4), containing the pump, the valve, the refilling unit, and also the control including the energy source.

The insulin-delivery units that have been described (summarised in Table 1) are open-loop systems. Insulin is delivered in accordance with profiles, the specific characteristics of which are evolved over several days of treatment with an artificial pancreas. The goal for the future must be a closed-loop system with a glucose sensor to measure the pertaining blood glucose concentration, which is compared with the normoglycaemia.

\section{Glucose sensor}

Intensive efforts are being devoted to the task of developing a miniaturised long-term stable and selective sensor for continuously monitoring the glucose level in blood or extracellular body fluids. The utilisation of a glucose sensor within an implanted closedloop artificial pancreas necessitates the conversion of the glucose concentration into an electric signal on the basis of an intra- or extracorporeal measurement. Without a doubt, the ultimate goal is a totally im- planted pancreas system incorporating a glucosesensing device. At present, glucose sensors are based primarily upon a variety of biochemical principles, while optical methods, such as polarimetry, have been virtually discarded. According to the requirements, there are systems in use which differ greatly in specificity, sensitivity, stability, handling and utilisation in autoanalysers.

The problems involved in the development of an implantable and miniaturised glucose sensor must be seen with respect to the situation that on the one hand there are at present, several clinically approved methods, while on the other, that not all of these methods lend themselves to implantation. To what extent future modifications to their operating mode or to components may eventually meet the requirements for implantation will be shown by further

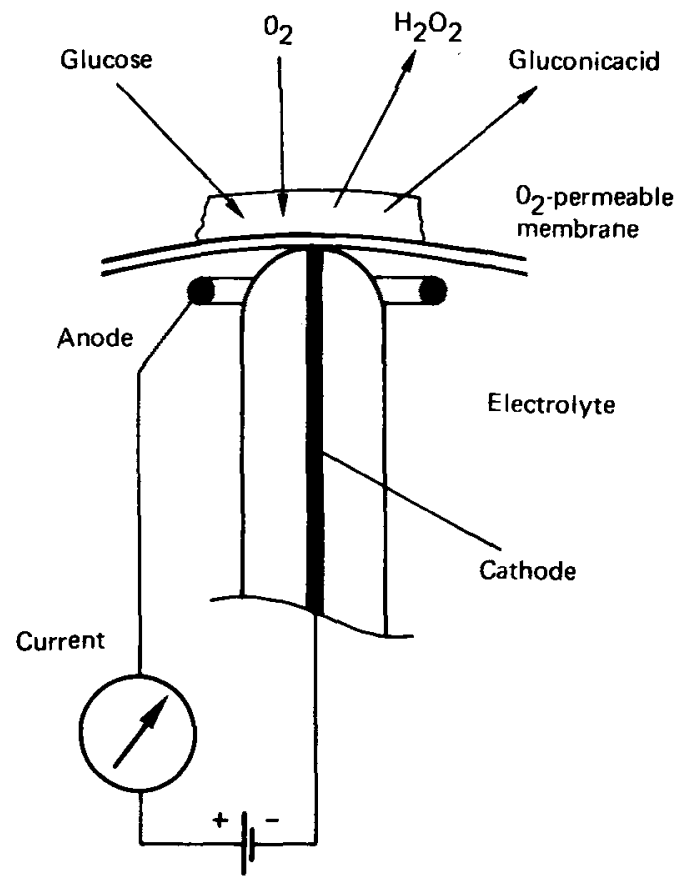

Fig. 5 Glucose enzyme-electrode with a Clark-type oxygen polarographic electrode (C. UPDikE, 1967)

Table 1. List of different implantable insulin-delivery systems

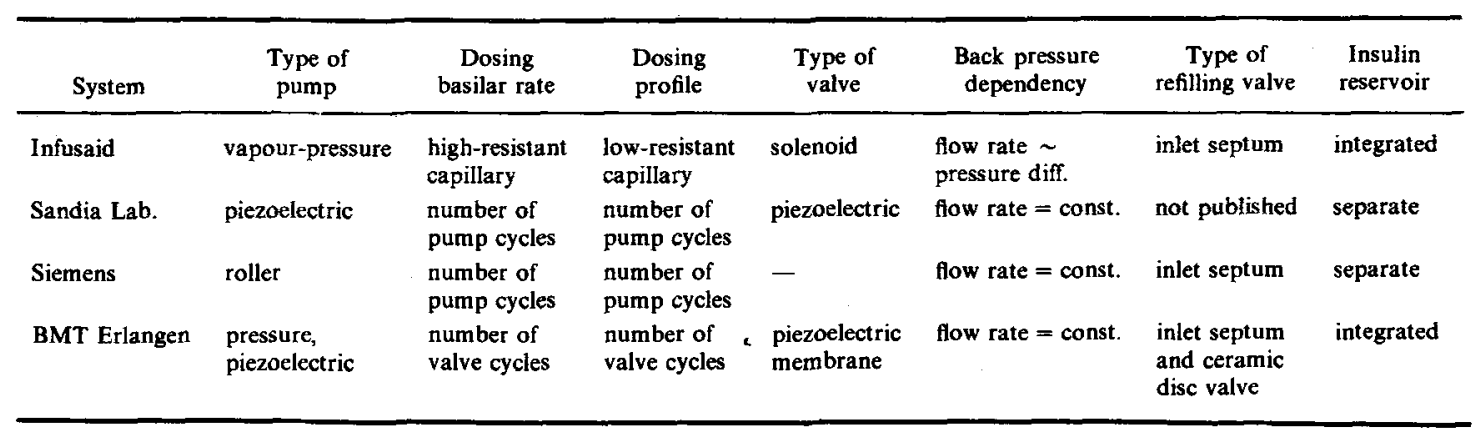


research work. If new principles have to be introduced, the problems will become even more difficult, because first the present state of performance has to be achieved, and, secondly, additional requirements as to size, energy consumption, longevity, biocompatibility etc. have to be met.

The present state of the art of sensing systems is characterised by a high level of overall performance and the systems are usually found incorporated in bedside devices coupled to an autoanalyser (Technicon, Miles) or, in the case of a closed-loop therapeutic control, to an insulin-infusion pump. These systems are based on the enzymatic oxidation of glucose by glucose-oxidase to gluconic acid:

$$
\text { glucose }+\mathrm{O}_{2} \stackrel{\text { GOD }}{\longrightarrow} \text { gluconic acid }+\mathrm{H}_{2} \mathrm{O}_{2}
$$

The determination of the glucose concentration is based indirectly on a second reaction: either (i) on colorimetric principles, where the formation of hydrogen peroxide determines the reaction rate of a colour indicator (ALBISSER, 1977) or (ii) the determination of either the oxygen depletion or the formation of hydrogen peroxide using a polarographic electrode covered by a membrane composed of the immobilised enzyme GOD (KADISH, 1969; UPDIKE, 1967; Clarke, 1977; Shults, 1979; BessmanN, 1973).

Up to date most of the systems measure blood glucose levels discontinuously, i.e. after withdrawal of blood and dilution with heparinised saline solution, the glucose concentration is determined by end-point or by kinetic calculations. With the Biostator of Miles Laboratories a continuous measuring device has been introduced (ClarKe, 1977). The immobilised glucose oxidase is attached to polyacrylamide and polycarbonate membranes. The functional service time has been reported to be between 8 and 140 h. BESSMANN et al. (1973) has verified the performance of an enzyme electrode under laboratory and in vivo conditions for up to 117 days. A limitation to long-term application to date is the irreversible loss of enzymatic activity and the stability of the enzyme/membrane compound. These effects are partially compensated in bedside systems by sophisticated recalibrating procedures.

At present, the hybrid enzyme/electrochemical sensor seems promising in bedside application. With the development of new methods and polymers for attaching and supporting the immobilised enzymes, such as with derivatised teflon membranes, an extracorporeal, wearable sensing unit might reasonably be expected in a matter of a few years (SHULTs, 1979).

As none of these systems promise short-term applicability in implantable insulin-delivery systems, there has been increasing concentration since the beginning of the seventies on electrochemical methods, in particular on the direct catalytical oxidation of glucose using metal electrodes (CHANG, 1973; GougH, 1977; GEBHARDT, 1978). From the evaluation of various designs of biofuel cell systems utilising glucose, it appears that platinum metal electrodes exhibit the best catalytic activity for the electrochemical oxidation reaction. Because these devices are composed of

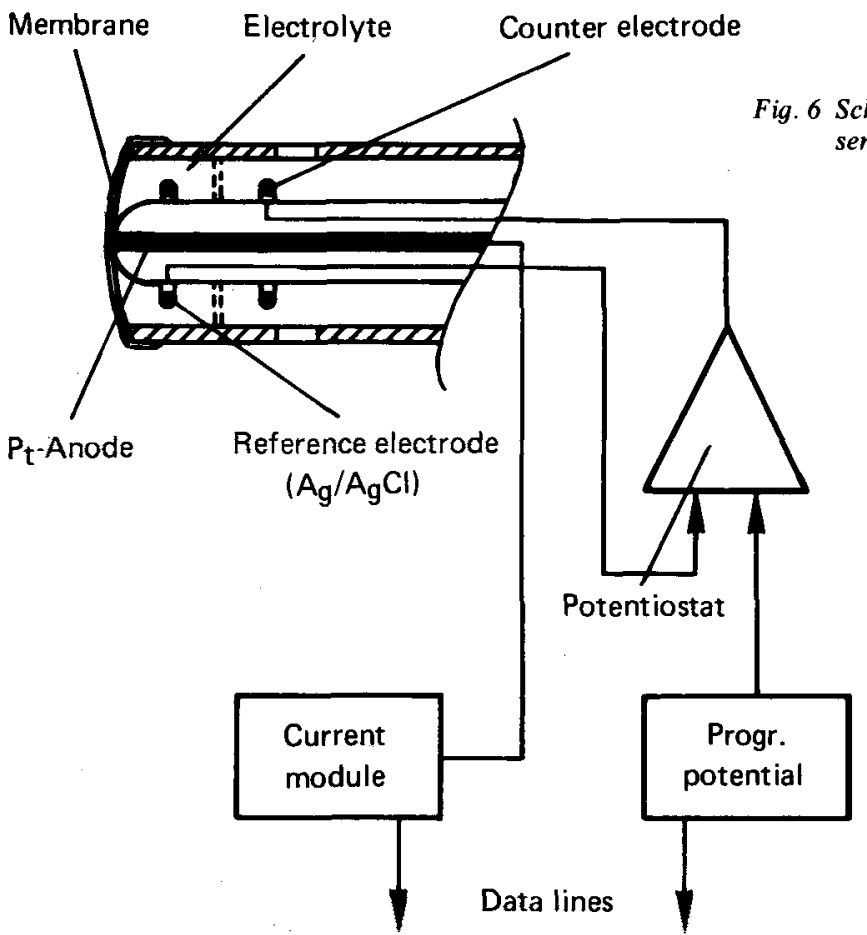


a nonconsumable metal anode, a counter electrode for the reduction of readily available species, such as oxygen, and an auxiliary reference electrode, they seem to be suitable for long-term application and have the potential for unlimited service life.

In principle, the reactions in a fuel-cell-type sensor are written for the anode:

$$
\mathrm{C}_{6} \mathrm{H}_{12} \mathrm{O}_{6}+\mathrm{H}_{2} \mathrm{O} \stackrel{\mathrm{P}_{1}}{\longrightarrow} \mathrm{C}_{6} \mathrm{H}_{12} \mathrm{O}_{7}+2 \mathrm{H}^{+}+2 \mathrm{e}^{-}
$$

and for the cathode:

$$
\frac{1}{2} \mathrm{O}_{2}+2 \mathrm{H}^{+}+2 \mathrm{e}^{-} \longrightarrow \mathrm{H}_{2} \mathrm{O} .
$$

Under the conditions of an ample supply of $\mathrm{O}_{2}$ and $\mathrm{H}_{2} \mathrm{O}$, the current of the anodic oxidation corresponds to the concentration of glucose at the metal surface. In general, it is assumed that the reaction proceeds via the formation of gluconolactone as an intermediate, to the stable product of gluconic acid in a two-electron oxidation step (ERNST, 1980). Results from LERNER et al. (1979) indicate that the reaction may approach complete oxidation to $\mathrm{CO}_{2}$ and $\mathrm{H}_{2} \mathrm{O}$.

This type of sensor acts as a transducer for the quantitative measurement of glucose in a diffusionlimited current mode. Since this sensor is relatively nonspecific, membranes have to be incorporated in order to minimise contributions from various monosaccharides, endogenous substances such as urea, proteins and amino acids (MARINCIC, 1979; RAO, 1978). Besides a lengthening of the response time due to the membranes, these interfering substances cause a deactivation of the platinum electrode. In addition, a decrease in performance may result from an insufficient supply of the cathodic fuel, i.e. oxygen, due to an unfavorable build-up of necrotic tissue surrounding the sensor. An evaluation of the tissueand blood-compatibility of the membranes and polymeric materials presently in use has to date not been of predominant interest.

The general attempt to overcome some of these problems has been to regenerate the electrode activity by short-duration pulses delivered via an auxillary electrode, thereby reduring the accumulated platinum surface oxides (Gough, 1977; GebHARDT, 1978). However, results have shown that the poisoning effects are not totally eliminated (RAO, 1978). Thus, the 'fuel cell' sensor seems to be out of the question for use as an implantable glucose sensor.

Research on different ways of operating the sensor electrode has initiated potentiostatic and potentiodynamic studies by involving the application of linear and triangular sweep voltages to a conventional three-electrode cell system (GebHARDT, 1978). The evaluation of the glucose oxidation currents at the platinum electrode is calculated from current/voltage curves. Results show that in the anodic and cathodic potential scan, characteristic 'peak' potentials can be attributed to the oxidation. From the almost logar- ithmic decrease of current with time at one of these 'peak' potentials it is concluded that the electrode surface is increasingly covered by some deactivating reaction products generated during the glucose oxidation process. In contrast to these potentiostatic studies there are decisive advantages in potentiodynamic measurements in which the potential is scanned continuously over the whole range from hydrogen to oxygen evolution. In this mode, the platinum surface is rejuvenated in every cycle, thus providing a reproducible surface state before starting the glucose measurement. Although the anodic 'peak' currents are strongly diminished in the presence of interfering substances such as urea and amino acids, the cathodic currents are only slightly inhibited. The contribution of the glucose may be evaluated from the measurement of the current at a prefixed cathodic potential under consideration of the approximated background current. In addition, the application of membranes with a rather low molecular cut-off point decreases the mass transport of interfering substances. The overall performance of the system shows a low dependency on interfering substances, even when the concentration changes within the physiological range.

Progress to an improved performance would be achieved when the exact partial current, which corresponds to the glucose contribution, could be subtracted unequivocally from the background currents of the platinum/platinum-oxide reactions. Further research will be focused on extending our knowledge as to the correlation of the glucose oxidation and the buildup and structural composition of the catalytic surface oxide layers. With more accurate information, an improved setting of the rejuvenating and measuring electrode potential is achievable, which must be seen as a prerequisite for reducing the deactivating processes and for enhancing the long-term stability of the sensing system.

The realisation of an implantable glucose sensor still depends upon the technological solution of a number of problems. Thus, it will be worthwhile looking for an intermediate sensor design which may be used in an extracorporeal application within a closed-loop implanted pancreas unit. A new approach for a noninvasive and continuous monitoring of the glucose concentration has been based on the well-known optical method of polarimetry (MARCH, 1979). The optical rotation of the aqueous humor of the eye is measured by a laser system integrated into a contact lens. Though several technological problems still remains to be solved, this system offers the advantage of recalibration or even replacement. The transfer of the glucose data to the implanted infusion system is accomplished by means of a telemetric communication system. Such systems have already been proven in pacemaker applications and are already available today. Thus, the hybrid system of an implanted dosing unit and an extracorporeal glucose sensor offers the possibility of realisa- 
tion in the near future and would indeed be of great significance in improving diabetic control. Thus, until an implantable sensor becomes available, it would seem reasonable to have an external sensor which may be incorporated into a closed-loop pancreas for regulation or into a pre- or patient-programmed pancreas for monitoring the effectiveness of the insulin infusion. Accordingly, the mode of operation of the sensor ranges from a rather fast and continuous to a slow and discontinuous response, thus eliminating a number of the prerequisites associated with implantable systems.

\section{Telecommunication system}

The purpose of the telecommunication system is to accomplish the exchange of programs and data between an external programming unit and the implanted control circuit (Fig. 7). In the case of an artificial pancreas connected to an implanted glucose sensor, the following interrogation pathways must be provided:

(i) To the implant

(a) Data transfer to the implanted control circuit to optimise the response and sensitivity settings of the sensor, and the algorithm of the transfer function of the glucose level and the patient-specific insulin requirement.

(ii) To the programming/monitoring unit

(a) Transfer of data back to the programmer to check the integrity of the transmission line. In addition, the validity of the data and their acceptance by the microprocessor is monitored at the same time.

(b) Transmission of data to monitor the momentary functional state of such parameters as battery voltage, glucose sensor signal, valve action etc.

(c) Monitoring of data that have been accumulated in separate registers, and which show, for example, the total amount of insulin delivered, or the variance of the glucose and insulin levels in a histogram, for a given time, or since replenishment of the insulin reservoir.

For the hybrid pancreas system, i.e. an implanted delivery unit controlled by an external glucose sensor, the interrogation pathways are as follows:

(i) To the implant

(a) Transfer of the glucose signal which already has been modified by the insulin delivery algorithm and adapted to drive the piezoelectric valves of the pump in an appropriate manner.

(ii) To the programming/monitoring unit

(a) Monitoring of the insulin delivery rate

The telecommunication program is quite different in the case of an open-loop system in which an insulin-delivery profile is preprogrammed and may be chosen by the patient in accordance with his needs. Monitoring of the glucose level at infrequent

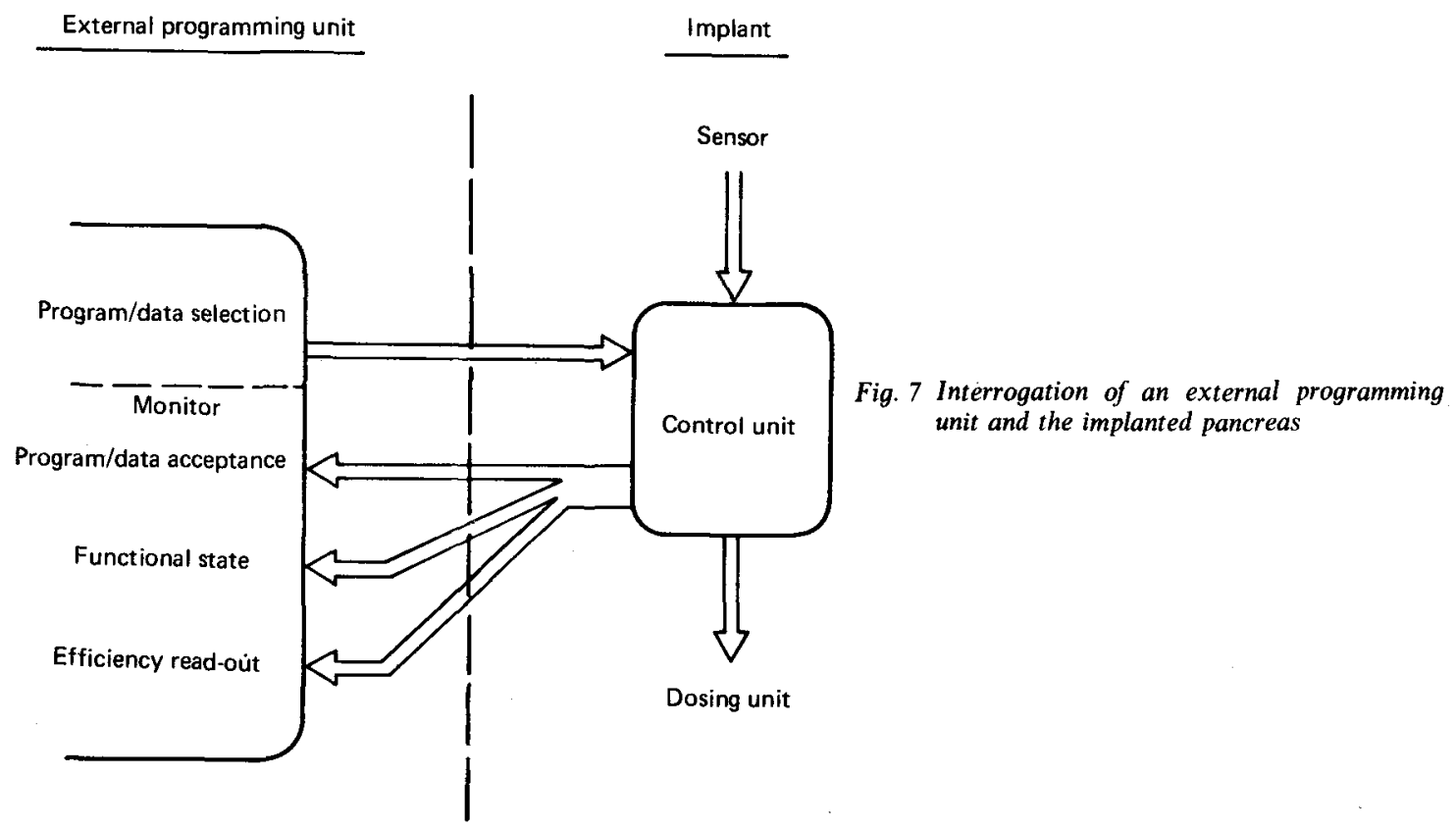


intervals has to be performed to check the effectiveness of the insulin profiles.

(i) To the implant

(a) The transmission line is generally used to initiate individual insulin profiles chosen by the patient.

(b) During and after the measurement of the glucose concentration, the telecommunication line carries corrected profile data whenever applicable.

(ii) To the programming/monitoring unit

(a) Data showing the type and frequency of the profiles chosen by the patient may be monitored to obtain the information needed for an improved matching of the insulin dose to the individual requirement.

Some of the principles of telecommunication systems, which may be utilised in the artificial pancreas, have already been well-proven in clinical pacemaker applications and are indicated in Table 2. Transmission of information with the aid of a static magnetic field is considered the simplest and oldest possibility. Pace- maker technology makes use of the same procedure, by the switching of a reed contact, to modify within the pacemaker the stimulation rate in several steps in the sense of an automatic program changeover. A disadvantage of the procedure is the indirect access to the desired frequency, since the individual values are called up only in a given fixed sequence, and the danger of an unitentional reprogramming by extreme static magnetic fields. The method of the rotating field is now a thing of the past; although considered reliable, it has the disadvantage of permitting modification of only a single pulse parameter.

A further method that is employed in connection with pacemakers utilised a pulsed magnetic field; it is used by a number of pacemaker manufacturers. The procedure permits the modification of several parameters; the pulse parameter to be called up is first coded as a digital word. The information is passed on by means of a reed contact which, in accordance with the two meanings of the letters of the word, is either opened or closed by the pulsed magnetic field. Communication is different from the form used with a pulse-code-modulated carrier frequency in that it is possible in only one direction and, since transmission

Table 2. Methods of telecommunication

\begin{tabular}{|c|c|c|}
\hline Telecommunication system & Transmitter & Receiver \\
\hline $\begin{array}{l}\text { Magnet field } \\
\text { static field } \\
\text { rotary field } \\
\text { pulsed field }\end{array}$ & $\begin{array}{l}\text { permanent magnet } \\
\text { rotary magnet } \\
\text { electromagnet }\end{array}$ & $\begin{array}{l}\text { magnetic switch } \\
\text { rotary magnet } \\
\text { magnetic switch }\end{array}$ \\
\hline $\begin{array}{l}\text { Carrier frequency } \\
\text { pulse code modulation }\end{array}$ & coil & coil \\
\hline $\begin{array}{l}\text { Ultrasonics } \\
\text { pulsed }\end{array}$ & piezocrystal & piezocrystal \\
\hline
\end{tabular}

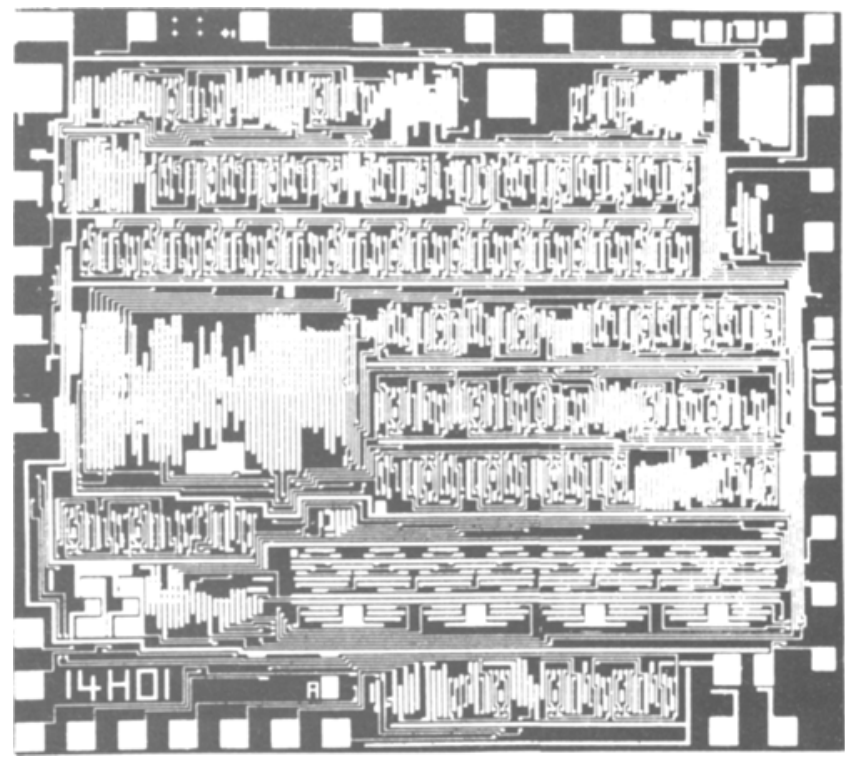

Fig. 8 Large-scale integrated circuit of the receiver/transmitter unit $(3.5 \times 4 \mathrm{~mm})$ 
makes no use of a discriminable carrier, it is more susceptible to interference. Communication with the implanted device by means of ultrasound makes use of the method employed in the remote-control of television sets and other electronic equipment. The method is considered rather unfavourable as the programming unit has to be placed directly on the skin of the patient to ensure reliable coupling-in in the biological milieu.

The particular advantage of pulse code modulation is its extreme insensitivity towards interference, which includes any unintentional program call-up by electric or magnetic interference fields in which the patient might move. The transmission of the programming instruction is effected with the aid of a radio-frequency carrier of $200 \mathrm{kHz}$, upon which the 8-bit word containing the information to be transmitted is modulated. In the artificial pancreas, the incoming signal is demodulated, and the program corresponding to the 8-bit word called up and translated into the appropriate function.

The requirements to be met by a complex transmitting/receiving circuit, suitable for a long-term implant, include low power consumption, high reliability, long-term stability and high circuit density to permit accommodation within the limited space of the control unit. State of the art technology is a hybrid assembly of a custom-integrated circuit. To achieve optimal adaptation of the interrogation circuit to the above mentioned requirements, it is neces-

\section{References}

Albisser, A. M., Leibel, B. S., Johnson, W., Denoga, A., Botz, C. K. and Maruiss, E. B. (1977) An improved technique for the rapid continuous measurement of whole blood glucose, suitable for clinical application in an artificial endocrine pancreas. Med. Progr. Technol., 5, 141-148.

Albisser, A. M., Jackman, W. S., Ferguson, R., Bahoric, A. and GorIYA, Y. (1978) A portable precision pumping system for chronic, programmed insulin infusion. Med. Progr. Technol., 5, 187-193.

Bessmann, S. P. and SchutZ, R. D. (1973) Prototype glucose-oxygen sensor for the artificial pancreas, Trans. Amer. Soc. Artif. Int. Oraans, 19, 361.

Blackshear, P. J., Rohde, T. D., Prosl, F. and BuchWALD, H. (1979) The implantable infusion pump: a new concept in drug delivery. Med. Progr. Technol., 6, 149-161:

Chang, K. W., Aisenberg, S., Soeldner, J. S. and Hiebert, J. M. (1973) Validation and bioengineering aspects of an implantable glucose sensor. Trans. Amer. Soc. Artif. Int. Organs, 19, 352.

Clarke, W. L., Santiago, J. V. (1977) The characteristics of a new glucose sensor for use in an artificial pancreatic beta cell, Artificial Organs, 1, 78.

Ernst, S., Heitbaum, J. and Hamann, C. H. (1980) The electrooxidation of glucose in phosphate buffer solutions: kinetics and reaction mechanism. Ber. Bunsenges. Phys. Chem., 84, 50-55. sary to concentrate the logic functions on a single chip, and to interconnect this 1.s.i. chip with the other components on a ceramic substrate. Although the costs involved are relatively high, the requirements of such a complex bidirectional data-transfer system could not have been met in a noninegrated design. Fig. 8 is a photograph of a $3.4 \times 4.0 \mathrm{~mm}$ chip, showing the high density of components and the bonding pads used to connect it to the input/output data lines.

\section{Concluding remarks}

At present, insulin infusion controlled by the actual blood-glucose level in a feedback system is possible only by the application of a bedside artificial pancreas or, less optimally, by a miniaturised extracorporeal pocket system. To increase reliability and convenience, especially for younger patients, an implantable insulin-infusion system is being developed. Some of the design components which are thought to be critical in long-term application have been discussed in detail. Engineering possibilities for controlling the insulin dose infused, the evaluation of new principles of measuring the glucose level, and the design of an interrogation system permitting monitoring and correction of the function of the implanted pancreas, have received special attention, since other problems involved are, to some extent, state of the art and already well-proven in pacemaker applications.

Franetzki, M., Prestele, K. and Funke, H. (1976) Gerät zur Infusion von Flüssigkeiten, Deutsche Offenlegungsschrift 2651962.

Gebhardt, U., Luft, G., Richter, G. and Sturm, F. von (1978) Development of an implantable electrocatalytic glucose sensor. Bioelectrochem. Bioenerg., 3, 607-624.

Gough, D. A., Aisenberg, S., Colton, C. K., Giner, J. and SOELDNER, J. S. (1977) The status of electrochemical sensor for in-vivo glucose monitoring. In: Blood glucose monitoring, Proceedings of a workshop-conference held in Freiburg (Germany) March 1976, Georg Thieme Publishers, Stuttgart, 10-21.

Kadish, H. A and Sternberg, J. C. (1969) Determination of urine glucose by measurement of rate of oxygen consumption, Diabetes, $18,467$.

Lerner, H., Giner, J., Soeldner, J. S. and Colton, C. K. (1979) Electrochemical glucose oxidation on a platinized platinum electrode in Krebs-Ringer solution. $J$. Electrochem. Soc., 126, 237-242.

MarCH, W., ENGermanN, R. and Rabinovitch, B. (1979) Optical monitor of glucose, Trans. Amer. Soc. Artif. Int. Organs, 25, 28-31.

Marincic, L., Soeldner, J. S., Giner, J. and Colton, C. K. (1979) Electrochemical glucose oxidation on a platinized platinum electrode in Krebs-Ringer solution: III Effect of urea., J. Electrochem. Soc., 126, 1687-1692.

RaO, J. R., Richter, G. J., Luft, G., Sturm, F. v. (1978) Electrochemical behaviour of amino acids and their influence on the anodic oxidation of glucose in neutral 
media, Biomat., Med. Dev. Artif. Org., 6, 127.

Schubert, W., Thull, R. and Schaldach, M. (1979) A modular implantable pancrease. 12th International Conference on Medical and Biological Engineering, Jerusalem.

Shults, M., Updike, S., Busby, M., Sokol, L. and GarbNER., C. (1979) Continuous in vivo glucose analysis based on immobilised enzyme bonded to derivatised teflon membrane. Trans. Am. Soc. Artif. Intern. Organs, 25, 66-70.

Spencer, W. J., Corbett, W. T., Dominguez, L. R. and
SHAFER, B. D. (1978) An electronically controlled piezoelectric insulin pump and valves. IEEE Trans., SU-25, 153-156.

SUMmers, G. B. (1970) A new and growing farnily of artificial implanted fluid devices. Trans. Amer. Soc. Artif. Int. Organs, 16, 218.

Thomas, L. J. and BessmanN, S. P. (1975) Prototype for an implantable micropump powered by peizoelectric disk benders. Trans. Amer. Soc. Artif. Int. Organs, 21, 516-520. UPDIKE, S. J. and HICKS, G. P. (1976) The enzyme electrode, Nature, 214, 986. 